\title{
Évolution et résonance du motif du jardin dans Henry VI, Richard II et Henry V
}

Jean-Christophe Mayer

\section{(2) OpenEdition}

Édition électronique

URL : http://journals.openedition.org/shakespeare/1294

DOI : 10.4000/shakespeare.1294

ISSN : 2271-6424

Éditeur

Société Française Shakespeare

Édition imprimée

Date de publication : 1 novembre 1995

Pagination : 65-75

Référence électronique

Jean-Christophe Mayer, «Évolution et résonance du motif du jardin dans Henry VI, Richard II et Henry V », Actes des congrès de la Société française Shakespeare [En ligne], 13| 1995, mis en ligne le 01 janvier 2007, consulté le 04 mai 2019. URL : http://journals.openedition.org/shakespeare/1294 ; DOI :

$10.4000 /$ shakespeare.1294 


\title{
SHAKESPEARE \\ LE MONDE VERT : RITES ET RENOUVEAU
}

Société Française Shakespeare

\author{
Actes du Congrès 1994 \\ sous la direction \\ de \\ M.T. JONES-DAVIES
}

Ouvrage publié avec le soutien de

BARCLAYS

BARCLAYS BANK S.A.

PARIS

LES BELLES LETTRES

1995 
Tous droits de traduction, de reproduction et d'adaptation réservés pour tous les pays.
(C) 1995 Société d'édition Les Belles Lettres, 95 bd Raspail 75006 Paris 


\section{Évolution et résonances du motif du jardin : Henry VI, Richard II, Henry V}

Peter Ure, dans son introduction au Richard II de l'édition Arden, met en évidence les sources de la scène dite du jardin de l'acte III, scène 4 . Il la conçoit comme l'aboutissement d'un motif récurrent dans les pièces historiques, mais n'en retrace pas l'évolution car tel n'est pas, bien entendu, son propos. Les sources sont, selon lui, de trois natures. L'influence de la tradition de la satire politique est sensible, de même que celle des sermons des prédicateurs médiévaux. Peter Ure souligne également l'utilisation du jardin dans les cérémonies et les spectacles tels que les masques. Du fait de ce triple héritage, la scène du jardin de Richard II reste empreinte d'une rhétorique quelque peu rigide et presque conventionnelle. Tout en prenant en compte ces analyses, peut-être faut-il aussi replacer le motif dans son évolution de Henry VI à Richard II et Henry $V$. Il serait vain cependant de prêter au dramaturge des desseins qui ne furent pas les siens. On suivra donc l'ordre de composition des pièces - et non l'ordre diégétique - dans le seul but de souligner la récurrence et la cohérence de l'image du jardin. Enfin, un réexamen du jardin de Richard II à la lumière des autres sources de cette imagerie devrait s'avérer fructueux et nous conduire à ne point omettre les résonances particulières du motif à l'époque élisabéthaine.

Dans 2 Henry VI et 3 Henry VI, si l'on considère la trilogie dans l'ordre de sa composition, le motif du jardin s'offre à nous comme quelques bribes de discours moralisateur emprunté à la tradition, celle qui ressortit aux sources évoquées par Peter Ure. On peut effectivement parler d'une rhétorique creuse, qui confine à l'allégorie, tant les différents protagonistes semblent mettre ce discours au service d'une cause qui est uniquement la leur. Il est frappant d'éprouver le caractère réversible - et donc sémantiquement vide - de ce discours. L'intrigante Reine Margaret, dans 2 Henry $V I$, met en garde le souverain contre son oncle, le Duc de Glocester, en ces termes: "Now 'tis the spring, and weeds are shallow-rooted ; / Suffer them now, and they'll o'ergrow the garden, / And choke the herbs for want of husbandry" (3.1.31-32) ${ }^{1}$. 
Ces conseils dissimulent mal le fait que la souveraine ellemême pourrait figurer aux côtés de ces "weeds". Elle tient en réalité un rôle de manipulatrice et la récurrence de l'adverbe "now" trahit quelque peu son empressement. Ces trois vers abritent, par ailleurs, des thèmes que l'on retrouvera plus tard dans Richard II notamment ou dans Henry $V$ : dans le jardin, représentation analogique du royaume, le passage du printemps (31) à l'été est vécu comme un instant de crise. Le printemps, synonyme de renouveau, apporte croissance et fertilité, deux notions qui n'ont pas en ellesmêmes de valeur positive, comme l'implique le verbe "overgrow" (32).

Richard Plantagenêt, dans cette même pièce, apprenant la perte des territoires français, fournit une autre instance de la réversibilité du motif du jardin : "Thus are my blossoms blasted in the bud, / And caterpillars eat my leaves away" (3.1.89-90). Richard s'approprie l'analogie jardin/royaume de la même façon qu'il souhaiterait faire sien ce même royaume. Mais cette fois le printemps n'a pas porté ses fruits, car l'ordre naturel s'est figé. Par le jeu d'un changement de perspective, Richard, le "jardinier" mécontent de 2 Henry VI, se métamorphose en plante dans 3 Henry VI par la magie du verbe enthousiaste du Comte de Warwick: "I'll plant Plantagenet, root him up who dares. / Resolve thee, Richard claim the English crown" (1.1.48-49).

Le motif s'est à nouveau inversé, saisissant l'opportunité onomastique du nom Plantagenêt. Richard est un homme, mais il est également un arbre, l'arbre généalogique de la Maison d'York qui, selon ses partisans, lui donne un droit légitime à la couronne. Déjà teinté d'un caractère emblématique, le motif prend des nuances encore plus marquées à l'acte II, scène 2 de 3 Henry VI. George, fils de Richard Plantagenêt, s'adresse en ces termes à la Reine Margaret: "But when we saw our sunshine made thy spring, / And that thy summer bred us no increase, / We set the axe to thy usurping root" (163-65). "Our sunshine" désigne bien évidemment l'emblème héraldique de la maison d'York tandis que "thy usurping root" fait resurgir l'image de l'arbre généalogique et insiste sur le pouvoir usurpé de la souveraine. Inversement, à l'acte III, scène 2 , l'arbre généalogique des York est lui-même mis en péril par un futur usurpateur. Richard, qui deviendra Duc de Glocester, ourdit ses intrigues dans le monologue qui clôt la scène. Il voue son frère, Edward, à l'exécration: "Would he were wasted, marrow, bones, and all, / That from his loins no hopeful branch may 
spring / To cross me from the golden time I look for" (125-27). L'arbre emblématique des York ne doit pas produire de ramification, de même que la nature ne doit pas suivre son cours si Richard veut parvenir à s'emparer du jardin d'Angleterre.

Mais ce n'est que dans1 Henry VI que l'on trouve le motif emblématique du jardin pleinement exprimé. Cette pièce fut, rappelons-le, une création postérieure au deux autres parties de la trilogie. La scène qui retient notre attention se déroule à Londres dans le jardin du Temple. La scène 4 de l'acte II constitue le seul exemple d'une scène de jardin dans les pièces historiques, avant celle de Richard II. Elle n'a, par ailleurs, aucun fondement historique, et s'offre donc comme le plus pur fruit de l'imagination du dramaturge.

Le jardin en question se distingue de l'image traditionnelle d'un lieu où l'on se retire pour goûter aux plaisirs du repos et de la méditation. Ce jardin du Temple se présente dès l'abord comme le lieu du secret qui permet à Richard Plantagenêt et à ses compagnons d'échapper aux oreilles indiscrètes. Le non-dit y règne également en maître. L'origine du débat qui anime les protagonistes n'est pas clairement énoncée par Richard dans les vers liminaires de la scène : "Great lords and gentlemen, what means this silence ? / Dare no man answer in a case of truth ?" (1-2)

L'objet du débat semble à tout le moins imprécis (notons le vague de l'expression "a case of truth") ; le jardin aide à préciser et à concrétiser l'enjeu du différend. Richard met en scène ce qui ressemble fort à un rituel emblématique : "Since you are tongue-tied and so loath to speak, / In dumb significants proclaim your thoughts" (25-26). Les "dumb significants" se présentent bientôt comme les roses rouges et blanches, emblèmes respectifs des maisons de Lancastre et d'York, que Richard enjoint ses compagnons de cueillir dans le but de manifester clairement leur appartenance à l'un où l'autre parti. On reconnaît tout le génie du dramaturge dans cette scène fictive qui situe les origines de la Guerre des Deux Roses dans un jardin emblématique, représentant par analogie le royaume d'Angleterre. Ajoutons qu'il s'agit du jardin d'une école de droit, celle du Temple, ce qui constitue indubitablement le lieu idéal pour une querelle portant sur les droits à la couronne et à ce jardin macrocosmique qu'est l'Angleterre.

Encore n'est-il question dans cette scène que d'un jardin tout juste ébauché. Dans Richard II, au contraire, le motif est dessiné de manière plus complète. Mais, dans cette pièce, le registre a 
changé ; l'emblématique se fait plus discret et cède la place à l'allégorie et à la parabole. Le motif, jusqu'alors synonyme de réversibilité (et donc moins signifiant), apparaît maintenant synonyme de renversement. Notons que la scène du jardin de Richard II (3.4) est historiquement fictive, tout comme celle de 1 Henry VI.

Conçu tout d'abord comme un lieu de refuge et de fuite pour l'épouse de Richard II, le jardin s'affirme rapidement comme un retour en plein coeur du réel, dans une scène qui se situe d'ailleurs au centre de la pièce. Ce retour s'effectue par le moyen indirect de l'allégorie. Rappelons tout d'abord le passage où l'analogie entre le microcosme du jardin et l'Etat anglais est établie par l'un des compagnons de labeur du jardinier :

Why should we, in the compass of a pale,

Keep law and form and due proportion,

Showing as in a model our firm estate,

When our sea-wallèd garden, the whole land,

Is full of weeds, her fairest flowers choked up,

Her fruit trees all unpruned, her hedges ruined,

Her knots disordered, and her wholesome herbs

Swarming with caterpillars?

(41-48)

La tirade repose intégralement sur l'analogie entre le "pale", l'enclos, et le "sea-wallèd garden". L'État anglais s'apparente au Hortus Conclusus, ce jardin clos d'héritage médiéval encore très en vogue aux époques élisabéthaines et jacobéennes ${ }^{2}$. L'analogie fait en outre écho aux termes du discours de Jean de Gand à l'acte II, scène 1. L'Angleterre y est décrite ni plus ni moins comme la terre d'élection de la nature qui peut, dans ce lieu fermé et protégé, retrouver sa pureté: "This other Eden, demi-paradise, / This fortress built by nature for herself ... / This happy breed of men, this little world" $(42-43,45)$. L'allusion aux "knots disordered", quant à elle, renvoit très certainement aux fameux "knot-gardens" qui jouirent d'une grande popularité sous le règne d'Henry VIII essentiellement $^{3}$. Les "wholesome herbs / Swarming with caterpillars" revêtent un intérêt particulier car l'expression introduit le rappel de la promesse de Henry Bolingbroke à l'acte II, scène 3 : "... Bushy, Bagot, and their complices, / The caterpillars of the commonwealth, / Which I have sworn to weed and pluck away" (164-66).

Bolingbroke a résolu de se faire le jardinier du royaume. Pourtant, c'est au roi qu'échoit normalement cette tâche. Le jardinier déplore qu'il n'en soit pas ainsi : "... O, what pity is it / That he had not so trimmed and dressed his land / As we this 
garden! ..." (56-58) En effet, dans l'ordre normal des choses, il est naturel - dans tout les sens de ce terme - que le roi veille à l'entretien du jardin : "Had he done so to great and growing men, / They might have lived to bear, and he to taste, / Their fruits of duty ..." (62-64).

Mais l'ordre naturel est en proie au tumulte; le roi ne dispose plus d'aucun contrôle sur la nature. Or, dans de nombreuses traditions, lorsque le roi n'a plus de pouvoir sur la nature, il n'est pas rare que l'on s'en défasse ou qu'on le remplace. L'anthropologue Luc de Heusch, spécialiste de la royauté sacrée, décrit ce phénomène :

Si le roi est une créature ambiguë, ambivalente, déculturée, c'est parce qu'il porte l'écrasante responsabilité de l'ordre naturel ... Dans un certain nombre de cas, le corps royal n'est utilisable à cette fin qu'aussi longtemps qu'il est parfait, intact. Dans d'autres, ce rouage essentiel doit être remplacé après un terme arbitrairement fixé, à moins qu'un sacrifice substitutif ne réalise l'indispensable régénération ${ }^{4}$.

C'est ce qui advient de façon allégorique dans cette scène, prélude aux bouleversements politiques de la suite de la pièce. Un nouveau jardinier, Henry Bolingbroke, s'apprête à congédier l'ancien, Richard II. Le motif s'inverse alors comme pour signifier le renversement prochain du souverain. L'ancien roi-jardinier est dépeint tel un parasite, un "wasteful King" (56), celui qui a vidé le royaume de sa substance en puisant dans les caisses de l'État. L'existence du roi-parasite est allégorisée dans les propos du jardinier: "He that hath suffered this disordered spring / Hath now himself met with the fall of leaf" (49-50). Le cycle des saisons devient la représentation analogique des cycles de l'histoire. L'image du roi s'inverse : il n'étend plus sa mainmise sur la nature mais se transforme en élément caduque de cette même nature. Il s'effeuille inexorablement comme le suggère "fall of leaf" (50). Le roi qui a trop végété pendant ces "idle hours" (67) devient un roi végétal qui se fane et s'affaisse ainsi que l'atteste la rime suivie "crown/down" (66-67).

La réaction outrée de la Reine au terme de la scène renforce l'aspect allégorique du passage tout en lui conférant une dimension biblique qui n'est point exempte d'une certaine ironie. Elle vitupère le jardinier, usant de termes, "Thou, old Adam's likeness, set to dress this garden" (74), qui opèrent insensiblement un rappel ironique. Le Roi, à l'instar de son jardinier, avait lui aussi la charge 
de l'entretien d'un jardin. Comme tout homme, mais aussi davantage que tout homme, du fait de sa fonction, il lui appartenait de respecter ce devoir biblique. Les propos de la souveraine font écho presque mot pour mot au célèbre passage de la Genèse : "And the LORD God took the man, and put him into the garden of Eden to dress it and to keep it" (II.15.) ${ }^{5}$.

Il est intéressant de noter, à ce propos, que la fin du XVI ${ }^{\mathrm{e}}$ siècle et le début du XVII ${ }^{e}$ coïncidèrent avec une période de vif débat sur l'origine et la nature du pouvoir politique. S'appuyant précisément sur les Saintes Écritures, nombreux furent ceux qui s'opposèrent aux théories catholiques ou protestantes qui proclamaient le droit des peuples à se rebeller contre un pouvoir tyrannique. Ces auteurs tentèrent d'imposer une conception patriarcale du pouvoir, le pouvoir royal trouvant sa légitimité dans celui d'Adam, le premier des patriarches. Ardent défenseur de la monarchie de droit divin, Sir Robert Filmer écrivit, probablement aux alentours des années 1640, un ouvrage au titre évocateur: Patriarcha / The Naturall Power of / Kinges / Defended against the / Unnatural Liberty / of the / People. Ce traité politique n'est en fait guère original dans son propos. Mais Filmer se révèle, en certains points, plus habile que ses prédécesseurs. Il précise par exemple qu'un roi peut être considéré comme ayant un pouvoir analogue à celui d'Adam et des patriarches bibliques. Il ne s'agit, pour Filmer, que d'une analogie et non d'un lien de descendance direct remontant à une hypothétique nuit des temps ${ }^{6}$. Ainsi le théoricien ne se place-t-il pas sur un terrain historique, ce qui rend par là-même ses arguments moins contestables et lui permet d'avancer la thèse suivante : "This lordship which Adam by creation had over the whole world, and by right descending from him the patriarchs did enjoy, was as large and ample as the absolutest dominion of any monarch which hath been since the creation"7.

Richard II n'est pas, loin s'en faut, un digne représentant de cette souveraineté de nature patriarcale. Il s'affirme, on l'a vu, comme un bien piètre Adam et un mauvais jardinier. De plus, ce médiocre patriarche porte la marque du meurtre originel de la pièce, celui du Duc de Glocester. C'est Bolingbroke en personne qui associe l'assassin du Duc à Caïn, le meurtrier biblique, à l'acte I, scène 1, vers 102-104. Mais Henry Bolingbroke, qui s'impose peu à peu comme le nouveau jardinier du royaume, a-t-il pour autant les qualités requises du patriarche biblique? En effet, au terme de la 
pièce, celui qui a dénoncé le meurtre originel est en fait lui-même marqué par la malédiction de Caïn, lorsqu'il bannit Exton, le meurtrier de Richard. "With Cain go wander through the shades of night", lui lance-t-il (V.6.43).

La scène du jardin cristallise donc beaucoup des tensions de la pièce et révèle toutes les ambiguïtés de la rivalité centrale, celle qui oppose Richard II à Bolingbroke ${ }^{8}$. Elle pose également une question fondamentale : un nouveau jardinier peut-il remplacer un mauvais Adam sans que le premier n'ait à souffrir la malédiction du second ? Pour répondre à cette question, il faut sans doute retourner aux Écritures. S'il est vrai que les rois descendent d'Adam, il est aussi écrit dans la Bible que Caïn fut le fondateur de la première cité. Abel, son frère, était pasteur et appartenait de fait à la Cité de Dieu, pour reprendre la terminologie de saint Augustin. Or, dans l'Angleterre d'après la Chute, il ne peut exister de jardinÉtat véritablement idyllique, car le Salut n'est pas dans la cité terrestre. Le politique, comme l'écrit saint Augustin, est voué à la chute, au péché :

Ainsi le premier fondateur de la cité terrestre fut un fratricide; vaincu par son envie, il tua son frère, citoyen de la cité éternelle et étranger sur cette terre. Il ne faut donc pas s'étonner que, bien plus tard, lors de la fondation de la ville destinée à prendre la tête de cette cité terrestre dont nous parlons, et à régner sur tant de nations, se soit reproduite une sorte d'image de ce premier exemplaire, cet "archétype ..." comme disent les Grecs 9 .

C'est l'éventuelle répétition de cette situation archétypale qui est en jeu dans le lien tracé par le jardinier entre le cycle des saisons et celui de l'histoire. Ainsi, la remontrance que la Reine adresse à son jardinier, "Dar'st thou, thou little better thing than earth, Divine his downfall ?" (79), laisse-t-elle entrevoir toute l'ironie du dramaturge. En effet, c'est bien parce qu'il est voisin des réalités de la glèbe ("little better thing than earth"), et donc du jardin, qu'à la différence des gouvernants, ce vieux jardinier a vu juste.

La scène s'est ouverte sur les paroles de la souveraine et de ses dames de compagnie, mais c'est bien sûr au jardinier que revient le dernier mot tant littéralement qu'au sens figuré. Lors de cette scène charnière, cet homme de la terre a planté efficacement le décor, celui de la chute allégorique, mais aussi bien réelle, de Richard II. Là où d'aucuns ont semé le désordre, lui sème "le re- 
pentir", la rue (106), cette herbe qu'il associe à "ruth" (107), la pitié.

L'allégorie se mêle au politique une dernière fois dans les pièces historiques. Cette reprise du motif du jardin se présente toutefois sur un mode mineur alors que le biais adopté diffère un tant soit peu. La scène 2 de l'acte $V$ de Henry $V$ contient une longue tirade que le Duc de Bourgogne destine au roi anglais. $Y$ figure derechef l'analogie qui associe le royaume au jardin, le Duc maniant l'allégorie avec conviction. Il décrit le royaume de France comme : "... this best garden of the world, / Our fertile France ..." (36-37). Le passage présente cependant une nouveauté en comparaison avec Richard II car il introduit la personification allégorique d'une idée abstraite. La Paix est cette idée qui tient une place centrale dans les propos du Duc: "Alas, she [la Paix] hath from France too long been chased, / And all her husbandry doth lie on heaps, / Corrupting in its own fertility" (38-40).

A la différence de Richard II, il est patent que les bouleversements qu'hérite tout jardin laissé à l'abandon n'apparaissent ici que pour mieux insister sur les vertus de la paix. Gageons que cette insistance sur les bienfaits de la paix civile, dans l'acte de résolution des conflits de la pièce, ne devait pas laisser indifférent à l'époque élisabéthaine. Ces allusions ne sont pas dépourvues de résonances. Roy Strong, qui a consacré un ouvrage au jardin anglais de la Renaissance, a montré les liens étroits qui unissaient l'image du jardin à celle de la paix élisabéthaine : "Gardens came to symbolize the Queen's peace and part of the cult of the Virgin Queen found its expression purely in horticultural terms.... The garden in short became Gloriana's glass". ${ }^{10}$

Dans le passage qui nous préoccupe, au contraire, se dessine une image destinée à frapper les imaginations : le jardin-royaume en proie à la sauvagerie la plus barbare est un lieu où l'humain et le végétal se confondent dans leur croissance anarchique :

Even so our houses and ourselves and children

Have lost, or do not learn for want of time,

The sciences that should become our country,

But grow like savages ... (56-59)

Cette description appelle étrangement son image inversée, celle du royaume policé comme aimait à la faire colporter Élisabeth Ière. Un extrait d'un traité sur le jardinage de 1578 en apporte la confirmation, en peignant cette fois la version officielle : 
"Blessed is the task, and worthy to bear thy name, O mighty Elizabeth, by whose moderation alone joyous Peace and her Olive so flourish in England that the British people may walk at random in the sunlight and gather in safety fragrant blossoms"11.

L'Angleterre paraît avoir recouvré son Age d'Or dans cette évocation. Henry $V$ offre en son terme une image semblable de l'Age d'Or retrouvé. Le Chœur, dont le discours clôt la pièce, a la charge de livrer la version officielle de l'histoire. Voici donc l'éloge du souverain éponyme: "This star of England. Fortune made his sword, / By which the world's best garden he achieved, / And of it left his son imperial lord" (Chœur, 6-8).

Cette épée que la Fortune lui a forgée est un des attributs de la personne du roi-guerrier, mais n'est-elle pas également en un sens l'outil du roi-jardinier? Grâce à ses qualités de "jardinier du royaume", Henry $\mathrm{V}$ a autorisé un retour, aussi éphémère fût-il, à un Eden de paix, "the world's best garden", expression faisant originellement référence à la France qui désormais s'applique, dans l'esprit des Anglais, au royaume d'Angleterre. L'Eden fut pourtant de courte durée, a tôt fait d'ajouter le Chœur. Sans doute faut-il entendre que le règne d'Henry ne fut qu'une parenthèse sans lendemain à la différence du paisible paradis élisabéthain, du printemps éternel que chantent les poètes tout entiers dévoués à la divine Elisabeth. Sir John Davies est de ces poètes; le troisième de ses Hymns to Astraea insiste précisément sur le caractère éternel de l'efflorescence du jardin d'Angleterre :

Earth now is greene, and heauen is blew,

Liuely Spring which makes all new,

Iolly Spring, doth enter;

Sweete yong sun-beames doe subdue

Angry, aged Winter.

Reserue (sweet Spring) this Nymph of ours

Eternall garlands of thy flowers,

Greene garlands neuer wasting;

In her shall last our State's faire Spring,

Now and for euer flourishing,

As long as Heaven is lasting ${ }^{12}$.

Il est peut-être loisible de déceler dans ces dernières évocations de l'Age d'Or - celle d'Henry $V$ comme celle des Hymns to Astraea - un certain messianisme. Henry Bolingbroke et Henry V sont des personnages qui possèdent, ou, comme on l'a vu, souhai- 
tent qu'on leur confère, certaines des caractéristiques du sauveur, ou du régénérateur du jardin d'Angleterre. Quant à Élisabeth Ière, les panégyriques cités ne sont que des exemples parmi d'autres d'un culte quasi messianique, orchestré de manière officielle, dont les instances sont légion. L'abondante littérature critique consacrée à la divine souveraine en témoigne ${ }^{13}$.

La royauté et la nature - même si cette dernière a surtout une valeur allégorique - paraissent donc étroitement liées dans les pièces historiques de Shakespeare, comme le prouve la récurrence du motif du jardin associé par analogie au royaume. Ce n'est pas tout à fait un hasard si le roi et le messie ont une fonction peu ou prou identique dans le monde naturel, comme l'a souligné Mircea Éliade : "Le Messie assume - sur un registre supérieur, évidemment - le rôle eschatologique du Roi-dieu ou du Roi-représentant de la divinité sur la Terre, et dont la principale mission était de régénérer périodiquement la Nature entière ... "14.

Le jardin ne forme qu'une partie de ce que l'on pourrait nommer la symbolique de la royauté, dans laquelle puise Shakespeare pour servir, bien entendu, ses propres desseins. Cette symbolique n'est pas seulement le fruit d'une tradition. La monarchie insuffle une vie nouvelle à la rhétorique allégorique traditionnelle et anime le développement de réseaux d'images qui mettent en valeur le pouvoir presque magique du souverain sur le monde naturel. L'analogie entre le roi et le jardinier demeure vivace à la fin du règne d'Élisabeth Ière et se conservera intacte jusqu'au règne de Charles Ier. Comme l'écrit Roy Strong, "The association of the new gardening with the principles of Divine Right is one of the most potent of all images. Gardening with its central theme of taming wild nature by the exercise of art, becomes an assertion of the royal will". ${ }^{15}$ Le jardin devient en quelque sorte une expression de l'absolutisme. Aussi n'est-il guère étonnant que le jardin en tant qu'attribut de la monarchie disparaisse avec la Guerre Civile pour ne renaître que difficilement après 1660 .

Jean-Christophe MAYER Centre d'Études et de Recherches Élisabéthaines Université Paul Valéry - Montpellier III 


\section{NOTES}

1. Toutes les citations des pièces de Shakespeare sont tirées de: William Shakespeare, The Complete Works, éds. Stanley Wells et Gary Taylor (Oxford: Clarendon Press, 1988).

2. Au sujet du Hortus Conclusus, voir Roy Strong, The Renaissance Garden in England (London: Thames and Hudson, 1979) 49.

3. A propos des knot-gardens, voir Strong 40.

4. Luc de Heusch, Écrits sur la royauté sacrée, Anthropologie sociale (Bruxelles : Éditions de l'Université de Bruxelles, 1987) 271.

5. King James Version.

6. Sur le caractère analogique de la descendance des rois chez Sir Robert Filmer, on pourra consulter : W. H. Greenleaf, Order, Empiricism and Politics, Two Traditions of English Political Thought 15001700 (London, New York et Toronto : Oxford U.P., 1964) 86-87.

7. Sir Robert Filmer, Patriarcha and Other Writings, éd. J. P. Sommerville (Cambridge, New York, Port Chester, Melbourne et Sydney: Cambridge U.P., 1991) 7.

8. Pour une discussion plus détaillée de la scène du jardin en rapport avec la rivalité Richard II-Bolingbroke, on lira avec intérêt : T. F. Berninghausen, "Banishing Cain: The Gardening Metaphor in Richard II and the Genesis Myth of the Origin of History", Essays in Literature 14 (1987):3-14.

9. Saint Augustin, La Cité de Dieu, CEuvres de Saint Augustin, trad. G. Combès, 4ème éd., vol. 36 (Paris, 1959) 47.

10. Strong 10.

11. Rembert Dodoens, A Niewe Herball, trad. Henry Lyte (London, 1578) III V . Cité dans Terry Comito, The Idea of the Garden in the Renaissance (Hassocks: Harvester Press, 1979) 19-20.

12. The Works in Verse and Prose of Sir John Davies, éd. A.B. Grosart (Fuller Worthies Library, 1869). Cité dans Strong 47.

13. Voir, entre autres, les ouvrages suivants : Roy Strong, The Cult of Elizabeth : Elizabethan Portraiture and Pageantry (London : Thames and Hudson, 1977); du même auteur, Gloriana: The Portraits of Queen Elizabeth (London: Thames and Hudson, 1987) ; Frances A. Yates, ASTREEE: Le symbolisme impérial au XVI siècle, trad. A. Huraut et J.-Y. Pouilloux, Littérature et Politique (Paris : Belin, 1989).

14. Mircea Éliade, Le mythe de l'éternel retour, Folio essais (Paris : Gallimard, 1969) 123-24.

15. Strong 200. 